\title{
Preface: logistics, optimization and transportation-in memory of the late Arne Løkketangen
}

\author{
Geir Hasle $^{1}$ • Lars Magnus Hvattum² . \\ David L. Woodruff ${ }^{3}$
}

Published online: 27 April 2017

(C) Springer Science+Business Media New York 2017

The Logistics, Optimization and Transportation (LOT) workshop honored the memory of Arne Løkketangen on September first and second, 2014, at Molde University College in Norway. The workshop name was chosen to comprise many of the research interests of Professor Løkketangen. There were 57 participants with presentations concerning new research in all three areas, which resulted in lively discussion.

It was the sort of workshop where Arne thrived. He joined Molde University College in 1988 after working for a decade as a consultant and software engineer. He received a $\mathrm{PhD}$ from the University of Bergen in 1995, and was promoted to the position of Professor in informatics at Molde College in 2001, where he worked until his untimely death on June 10, 2013. His research continues to have an impact on the workshop topics.

This issue is a product of that workshop. Many of the papers come from workshop attendees directly, and others were submitted by authors working on these topics but unable to attend. A total of 30 papers were submitted to the special issue, out of which 11 were accepted.

"Assessing policy quality in a multistage stochastic program for long-term hydrothermal scheduling," by de Matos, Morton, and Finardi, goes to the heart of the issue with stochastic programming: how good is the solution with respect to the stochastic problem itself? Most research in this area focuses essentially on solving a deterministic version of the problem. In this paper, the authors explore using multiple scenario trees versus using a single scenario tree. They also compare naive Monte Carlo sampling, randomized quasi Monte Carlo sampling, and Latin hypercube sampling. The work is done in the context of long-term hydro scheduling.

In "Optimizing high-level nuclear waste disposal within a deep geologic repository," Johnson, Newman, and King consider the problem of placing nuclear waste in deep geologic repositories. The authors test three different objective functions that minimize the heat load

Geir Hasle

Geir.Hasle@sintef.no

1 Mathematics and Cybernetics, SINTEF Digital, Oslo, Norway

2 Faculty of Logistics, Molde University College, Molde, Norway

3 Graduate School of Management, University of California Davis, Davis, CA 95616, USA 
concentration. Symmetry reduction constraints, perturbations, and heuristics are used to solve larger problem instances.

"A dynamic discrete network design problem for maintenance planning in traffic networks," by Fontaine and Minner, looks at a bi-level problem where the top level determines which road in a network to maintain and the second level optimizes the path through the network. Karush-Kuhn-Tucker conditions are used to transform the model so that it can be solved by Benders decomposition. Computational experiments demonstrate the efficacy of the algorithm.

"A path-based capacitated network flow model for empty railcar distribution," by Heydari and Melachrinoudis, studies the problem of assigning empty railcars to customer demands. The authors present a new, rich formulation for the problem that considers important technical and business requirements. The result of a realistic test instance shows an $18 \%$ improvement in the objective function, when compared to a model currently used by the US railroads.

In "Combined ship routing and inventory management in the salmon farming industry" Agra, Christiansen, Ivarsøy, Solhaug, and Tomasgard deal with a maritime inventory routing problem rooted in the operations of a large salmon farming company. Model reformulations and valid inequalities are presented to improve on a mixed integer linear programming formulation. To cope with realistically sized instances, the authors exploit practical aspects of the problem and create a matheuristic.

"Improved solutions to dynamic and stochastic maritime pick-up and delivery problems using local search," by Tirado and Hvattum, presents improved heuristic methods for a routing and scheduling problem arising in industrial shipping. Uncertainty in future demands is represented in the form of scenarios, and the dynamic nature of the problem is handled in a simulation framework. The authors improve on previous heuristics by exploiting the information from the scenarios within local search methods.

"Vessel capacity restrictions in the fleet deployment problem: an application to the Panama Canal," by Herrera, Agrell, Manrique-de-Lara-Peñate, and Trujillo, contains an analysis of the consequences following the expansion of the Panama Canal. By using a linear fleet deployment model, the authors find that using larger vessels will lead to lower vessel costs, higher utilization rates, and lower bunker fuel consumption after the canal capacity has increased from 5000 to 13,000 TEUs.

"A POPMUSIC-based approach for the berth allocation problem under time-dependent limitations," by Lalla-Ruiz, Voß, Expósito-Izquierdo, Melián-Batista, and Moreno-Vega, fits perfectly with the themes of the LOT workshop that inspired this issue and where the second author was a plenary speaker. The paper makes use of a meta-heuristic to provide solutions to an important problem in logistics related to scheduling ships with limited berthing positions and water depth constraints.

"Multi-trip pickup and delivery problem with time windows and synchronization," by Nguyen, Crainic, and Toulouse, describes a tabu search meta-heuristic for a rich problem in logistics. The problem combines vehicle routing and scheduling and the paper describes a new optimization algorithm that is competitive with the state of the art for related problems. This paper describes the first test instances of this particular problem.

The papers "A cluster-first route-second approach for the Swap Body Vehicle Routing Problem," by Miranda-Bront, Curcio, Méndez-Díaz, Montero, Pousa, and Zabala, and "A relax-and-repair heuristic for the Swap-Body Vehicle Routing Problem" by Absi, Cattaruzza, Feillet, and Housseman, consider the same problem. The Swap Body Vehicle Routing Problem is variant of the Truck and Trailer Routing Problem-a generalization of the classical VRP. It was introduced by the German company PTV and the EURO Working Group on Vehicle Routing and Logistics optimization-VeRoLog_in connection with the 2014 VeRoLog 
Solver Challenge. The two papers originate from the work of finalist teams in the Challenge. Miranda-Bront et al. propose a cluster-first route-second heuristic, whereas Absi et al. investigate a relax-and-repair heuristic embedded in a population-based heuristic. Both algorithms yield excellent results on the test instances provided, and they have potential to be applied in practice. 